\title{
Coordinate Signal Control in Urban Traffic of Two-direction Green Wave based on Genetic BP Neural Network
}

\author{
Shaojiao Lv ${ }^{1, a}$, Chungui $\mathrm{Li}^{2, \mathrm{~b}}$, Zheming $\mathrm{Li}^{1}$ and Qingkai Zang ${ }^{1}$ \\ ${ }^{1}$ School of Electrical and Information engineering, Guangxi University of Science and Technology, \\ Liuzhou 545006, China \\ ${ }^{2}$ School of Computer Science and Technology, Guangxi University of Science and Technology, \\ Liuzhou 545006, China
}

alsj861209@163.com, ${ }^{\mathrm{b}}$ Icg6881@tom.com

Keywords: Coordinate signal control; Genetic algorithm; BP neural network; Green wave bandwidth.

\begin{abstract}
To maximize the bandwidth of green wave of trunk road is a main issue in the research of signal control in urban traffic. However, the traditional analytical algorithm can not be applied in actual traffic widely. A novel dynamic two-direction green wave coordinate control strategy was proposed to overcome the problem. By combining the genetic BP neural network with the traditional analytical algorithm, the urban traffic of two-direction was controlled coordinately online. Finally, an actual example was presented. It shows that not only the green wave bandwidth, the phase-difference of each intersection and the critical cycle of trunk road were optimized according to real-time traffic flow, but also our algorithm can be used in different traffic condition by adjusting the parameters of the model.
\end{abstract}

\section{Introduction}

With the city traffic jam becoming more and more serious, the coordinate control in the urban road of green wave became the researching focus which was an important selection of the priority control mode of urban traffic signal control system.

While the researches about the coordination control in urban traffic of two-direction green wave were mainly MAXBAND [1] and MULTIBAND [2], Paper [3] introduced the traditional algorithm including graphic method and analytic method. Many investigations have been carried out by Chinese scholars. In order to overcome the problem that traditional analytical algorithm can not be put in practical application, Lu [4] and Wang [5] pointed out modified ideas to get a new traditional analytical algorithm method. Although the algorithm realized the object of optimized line control, it had defects in accuracy because it still depended on decisive mathematical model to treat the uncertainty traffic signals. A number of intelligent control methods have been introduced into this field. Chang [6] proposed a directly optimize design method employing genetic algorithm to reduce the delay system. Shou [7] proposed a coordinate control theory based on group decision-Making. Following minimum delay time principle, Shen [8] proposed a trunk control algorithm based on fuzzy theory and neural network algorithm. Kong [9] proposed a dynamic two-way green wave coordination control strategy based on multi-agent.

On one hand, though the intelligent control algorithm can adapt to the complicated system, the stability and control precision of intelligent algorithm still need to be improved. On the other hand, traditional algorithm needs a long time to design the required parameters according to the real-time parameters of green ratio, the space of each intersection, the initial cycle. In order to solving these problems, a algorithm combining the advantages of traditional analytical algorithm with intelligent control algorithm was pointed out in the paper, based on previous research work and the genetic back propagation (BP) neural network algorithm. Furthermore, the presented algorithm can realize the dynamic adjustment in real time. 


\section{The Traffic Flow Models}

The geometric model. In order to contrast with the traditional analytical algorithm in Paper [4] and paper [5], this paper used the same road model as figure 1. There are eight intersections and the distances between the adjacent intersections are $350 \mathrm{~m}, 400 \mathrm{~m}, 160 \mathrm{~m}, 540 \mathrm{~m}, 280 \mathrm{~m}, 270 \mathrm{~m}$, the initial common cycle is $80 \mathrm{~s}$, the velocity is $11 \mathrm{~m} / \mathrm{s}$. (From west to east direction is downward and from the east to west direction is upward).

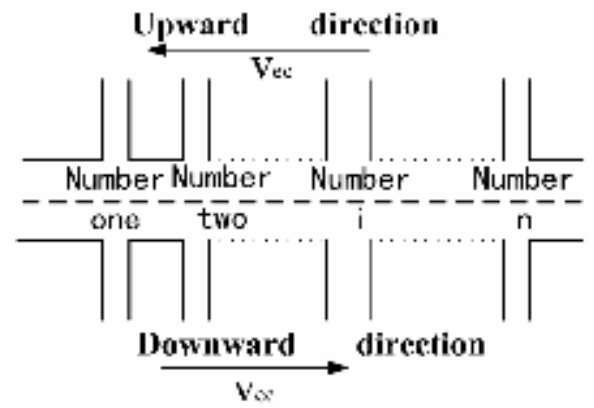

Fig.1 Sketch map of urban arterial traffic

The optimization criterion. Coordinate signal control in urban traffic of two-direction green wave is based on passing the traffic trunk road with no stop at the velocity of green wave. It can relieve the traffic congestion effectively. The optimization criterion of this paper is getting the largest through-width at the certainly velocity.

The influence factors. Green ratio, velocity, signal cycle and distance between adjacent intersections are the major factors need to be considered in coordination control.

\section{Genetic BP Neural Network}

Uses of genetic BP neural network. Any nonlinear mapping can be achieved from input to output through BP neural networks treating multivariate problems. Through training samples, it could determine the network structure, learn parameters, and establish the nonlinear relationship between the output and the input. The network weights dependent on the effects of learning extraordinarily because of the BP neural network used gradient descent method. It is tend to be premature and thus converge in local minimum value. Genetic BP neural network used the advantages of the genetic algorithm whose search performance is highly parallel, random and adaptive. The proposed Genetic BP neural network is realized by coding the weights and threshold of BP neural network as chromosome, and then those chromosomes are put in genetic algorithm for selection, crossover and mutation.

Designs of Genetic BP neural network. The influence factors such as green ratio, velocity, signal cycle and the distance between adjacent intersections are input while the value of factors such as phase-difference, the common cycle and the bandwidth of green wave are output, as Fig.2.

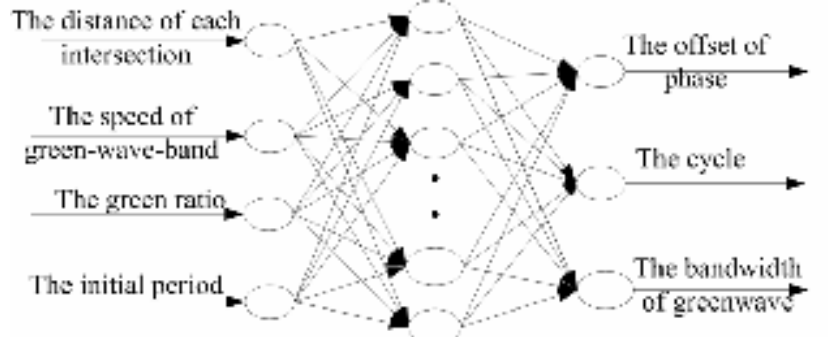

The imput layer The hidden layer The output layer

Fig.2 The topological structure of network

Generally, if we use the BP neural network to simulate a set of known data, we must train the neural network by using the known input -output data as learning samples. The enumerated method software is used to realize the method of traditional analytical algorithm for data collection platform. 900 sample data were gained by using enumeration method. We choose the front 600 sets of data as training sample and the later 300 sets of data were used for testing. The data were reliable and widely covering 
because it is not only referenced the principle of the largest migration of green ratio to be minimum but also considered the influence of the minimum green ratio's bottleneck restriction for green wave bandwidth. Therefore, using the sample to train the BP neural network which has the very strong nonlinear mapping ability can approach any complex nonlinear mapping relationship of input-output.

\section{The Experiment and analysis}

MATLAB 2009a neural network toolbox was used in this paper, there are three simulated results of the cycle, the green wave bandwidth and the difference of phase, as figure 3, figure 4, figure 5 . Obviously, the results of the algorithm proposed here was better than the traditional analytical algorithm, it can improve the output of the system quickly according to the real-time traffic flow data at the same condition, as fig. 1. The comparison of the two algorithms is shown in Table 1. We can see that the bandwidth obtained by our algorithm is $28 \%$ while that got by Lu [4] is $33 \%$. Even though our algorithm wasn't get wider bandwidth, it approached the complex nonlinear mapping relationship of the traditional analytical algorithm. If you give a group of input into the trained network, it can output immediately compared with the traditional analytical algorithm.

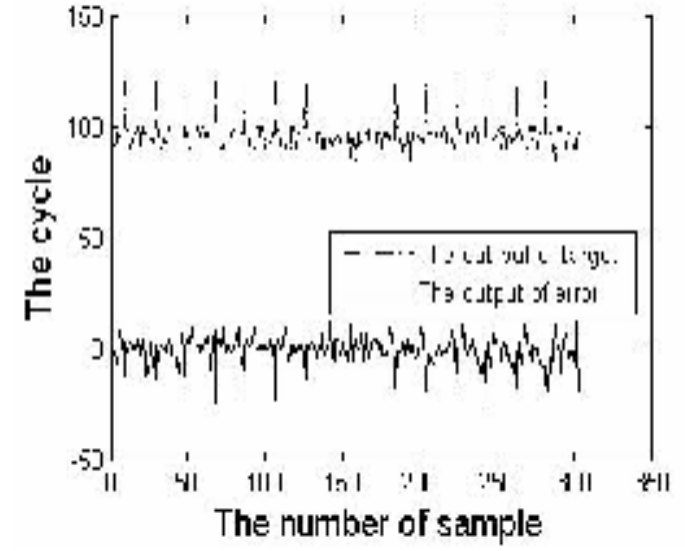

Fig.3 The simulation results of the cycle

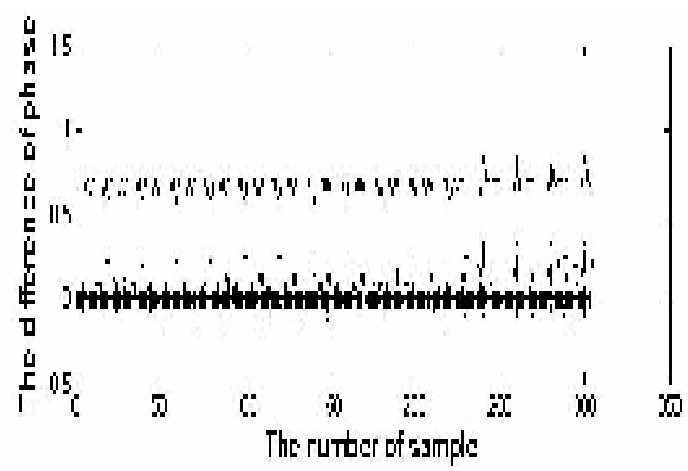

Fig.5 The simulation results of the difference of phase

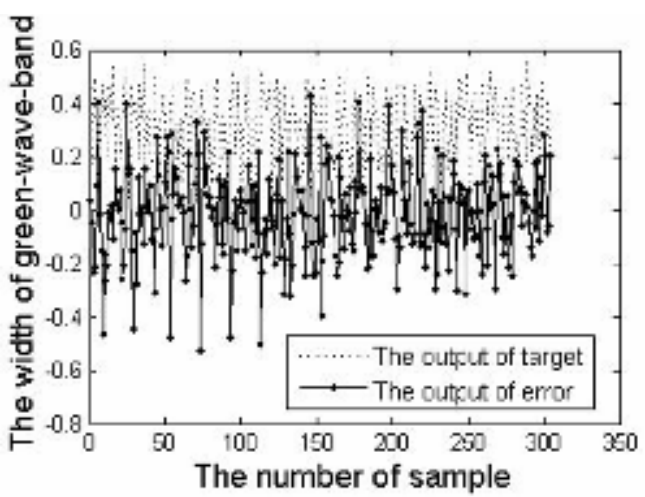

Fig.4 The simulation results of the width of green-wave band

Table 1 The comparison of the two algorithms

\begin{tabular}{|c|c|c|}
\hline & $\begin{array}{c}\text { The Lu's } \\
\text { algorithm }\end{array}$ & $\begin{array}{c}\text { The new } \\
\text { algorithm }\end{array}$ \\
\hline Critical cycle & 80 & 82 \\
\hline Bandwidth & $33 \%$ & $28 \%$ \\
\hline Phase difference.1 & 75 & 72.5 \\
\hline Phase difference. 2 & 15 & 20 \\
\hline Phase difference.3 & 65 & 67.5 \\
\hline Phase difference.4 & 83 & 67.5 \\
\hline Phase difference.5 & 20 & 20 \\
\hline Phase difference.6 & 62 & 67.5 \\
\hline Phase difference.7 & 71 & 65 \\
\hline Phase difference. 8 & 18 & 25 \\
\hline
\end{tabular}

\section{Conclusions}

The algorithm proposed here can deal with many problems. It can be used in different traffic road condition by adjusting parameters and changing the number and size of distance and green ratio. Furthermore, it could improve the reliability and practicability of the system in real time, so we could use the algorithm according to the actual traffic flow to adjust dynamically online under any traffic situation. 


\section{Acknowledgements}

This paper is supported by Guangxi Scientific Research and Technology Development Foundation (0992006-13).

\section{References}

[1] Little J. DC.: Operations Research, Vol. 14(1966), p.568.

[2] N. H. Gartner, ASSMANN S. F., LASSAGA F.: Transportation Research B, Vol. 25(1991), p.55.

[3] Editorial Committee of Traffic Engineering Manual of Highway and Transportation Society of China: Traffic Engineering Manual (People's Communications Publishing House, china 1998.

[4] K. Lu. Research on the Foundational Theory and Key Techniques of coordinate Signal control in urban Traffic Network (Ph.D., South China University of Technology, China 2010), p. 51.

[5] D.H. Wang, X.R. Yang, X.M. Song: Journal of Ji Lin University (Engineering and Technology Edition, Vol41 (2011) No.1, p.29.

[6] Y.T. Chang, G.X. Peng: Journal of Traffic and Transportation Engineering, Vol. 3(2003) No.2, p.106.

[7] Y.F. Shou: Theory and Method Research of Wide-area Traffic Signal Coordinated Control based on Group Decision-making. [D] (Ph.D., South China University of Technology, China 2011), p. 32.

[8] G.J. Shen, Y.X. Sun: Systems Engineering Theory and Practice, Vol. 24(2004) No.3, p.99.

[9] X.J. Kong, G.J. Shen, Y.X. Sun: Journal of PLA University of science and technology (Natural science edition), Vol. 11(2010) No.5, p.544. 